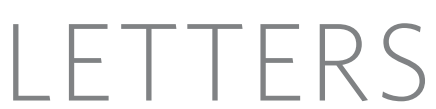

\title{
Neurite arborization and mosaic spacing in the mouse retina require DSCAM
}

\author{
Peter G. Fuerst ${ }^{1}$, Amane Koizumi ${ }^{2} \uparrow$, Richard H. Masland ${ }^{2} \&$ Robert W. Burgess ${ }^{1}$
}

To establish functional circuitry, retinal neurons occupy spatial domains by arborizing their processes, which requires the selfavoidance of neurites from an individual cell, and by spacing their cell bodies, which requires positioning the soma and establishing a zone within which other cells of the same type are excluded ${ }^{1}$. The mosaic patterns of distinct cell types form independently and overlap. The cues that direct these processes in the vertebrate retina are not known ${ }^{2,3}$. Here we show that some types of retinal amacrine cells from mice with a spontaneous mutation in Down syndrome cell adhesion molecule (Dscam), a gene encoding an immunoglobulin-superfamily member adhesion molecule ${ }^{4,5}$, have defects in the arborization of processes and in the spacing of cell bodies. In the mutant retina, cells that would normally express Dscam have hyperfasciculated processes, preventing them from creating an orderly arbor. Also, their cell bodies are randomly distributed or pulled into clumps rather than being regularly spaced mosaics. Our results indicate that mouse DSCAM mediates isoneuronal self-avoidance for arborization and heteroneuronal self-avoidance within specific cell types to prevent fasciculation and to preserve mosaic spacing. These functions are analogous to those of Drosophila DSCAM (ref. 6) and DSCAM2 (ref. 7). DSCAM may function similarly in other regions of the mammalian nervous system, and this role may extend to other members of the mammalian Dscam gene family.

We have identified a spontaneous mutation in mice that creates a loss-of-function allele of Dscam, the Drosophila homologues of which function in both isoneuronal self-avoidance for dendrite arborization and heteroneuronal self-avoidance for axon tiling ${ }^{7-12}$. The recessive mutation arose in the $\mathrm{BALB} / \mathrm{cByJ}$ genetic background and caused an overt neurological phenotype. Mutant and wild-type mice are indistinguishable at birth, but are severely uncoordinated by postnatal day 3 (P3); as adults, the mutant mice have spontaneous seizures and kyphosis, but are fertile and long-lived ( $>24$ months; see http://www.jax.org/research/media/wild_type.html for video). Through positional cloning (see Methods), a mutation was identified in Dscam. Sequencing of genomic and complementary DNA from affected mice revealed a 38-bp deletion in exon 17 , causing a frame shift resulting in ten unique amino acids followed by a premature stop codon (Supplementary Fig. 1). This mutation truncates the protein in the second fibronectin repeat (Fig. 1a). Dscam messenger RNA levels in the brain were reduced by $70 \%$ in affected mice, consistent with nonsense-mediated decay (Fig. 1b).
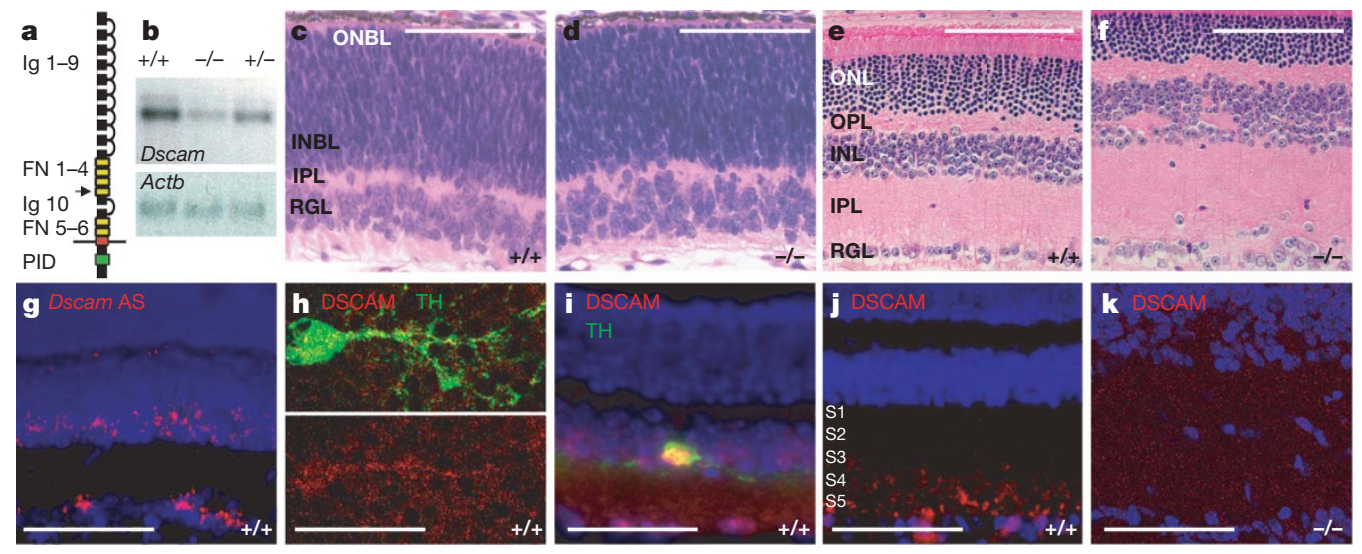

Figure 1 | Identification of a mouse Dscam mutation. a, A schematic of the DSCAM protein domain structure. The extracellular portion of DSCAM consists of ten immunoglobulin-like repeats (Ig) and six fibronectin domains (FN). The Dscam deletion truncates the coding sequence in the second fibronectin domain (arrow), which is before the transmembrane and PAK1-interacting domains (PID). b, Northern blotting of mRNA purified from whole brains of wild-type $(+/+)$, Dscam $^{+/-}$and Dscam $^{-/-}$mice revealed a $70 \%$ reduction in Dscam mRNA in the mutant sample. $\beta$-actin $(A c t b)$ was used as a loading control. c-f, Haematoxylin and eosin stained sections of $\mathrm{Dscam}^{-/-}$and wild-type retinas from P0 and adult mice. The $\mathrm{Dscam}^{-/-}$retina is indistinguishable from that of the wild type during embryonic stages of development and at birth $(\mathbf{c}, \mathbf{d})$. INBL, innerneuroblast

layer; ONBL, outerneuroblast layer; ONL, outernuclear layer; OPL, outerplexiform layer. e, f, In the adult Dscam $^{-1-}$ retina, the inner nuclear (INL), inner plexiform (IPL) and retinal ganglion (RGL) layers are disorganized compared to those of the wild-type retina, whereas other retinal layers are indistinguishable from that of the wild type. g, In situ hybridization with Dscam antisense probes (Dscam AS) revealed expression in a subset of cells in the inner nuclear and ganglion cell layers. $\mathbf{h}$, Whole control P15 retina stained with antibodies to DSCAM and TH. i, A section of a control adult (6-10-week-old) retina labelled using antibodies to DSCAM and TH. $\mathbf{j}$, A section of wild-type P15 retina stained with DSCAM antibodies. k, A section of the $D_{\text {scam }}{ }^{-/-}$retina stained with an antibody to DSCAM. Scale bars: c, d, $80 \mu \mathrm{m}$; e, f, $106 \mu \mathrm{m} ; \mathbf{g}, 160 \mu \mathrm{m}$; h, $45 \mu \mathrm{m}$; i, $120 \mu \mathrm{m}$; j, k, $65 \mu \mathrm{m}$. 
Despite the overt neurological phenotype of $\mathrm{Dscam}^{-/-}$mice, examination of the central nervous system of adults and developing embryos did not reveal any gross disorganization, with the exception of a caudal folium of the cerebellum (Supplementary Fig. 2), implying a subtle phenotype or a subpopulation of affected cells.

We did find disorganization of postnatal retinal anatomy (Supplementary Fig. 3). At birth or before, the mutant retina was indistinguishable from that of control mice (Fig. 1c, d). However, by P4, the ganglion cell layer and developing inner plexiform layer (IPL) were disorganized (not shown). This disorganization persisted into adulthood in amacrine and ganglion cells (Fig. 1e, f), and was confirmed with marker analysis (Supplementary Fig. 4). Within these cell populations, many subtypes are present ${ }^{13,14}$, and Dscam was expressed in a subset of cells in these layers (Fig. 1g). Double in situ hybridization with markers of defined amacrine subtypes indicated that Dscam was expressed in dopaminergic amacrine cells (tyrosine hydroxylase (Th)-positive) and in bNOS-positive (Nos1-expressing) cells, but not in choline acetyltransferase (Chat)-positive starburst amacrine cells (SACs) or disabled 1-positive (Dab1) AII amacrine cells (Supplementary Fig. 5). Immunofluorescence with an antibody against the extracellular domain showed that DSCAM completely overlapped dopaminergic amacrine cell staining in $>400$ cells examined. In whole-mount retinas at P15, anti-DSCAM staining was distributed across the soma and processes of dopaminergic amacrine cells, but redistributed to the cell body in the adult retina (Fig. 1h, i). Additional immunoreactivity was detected in retinal cross-sections in lower, more vitreal layers of the IPL, below the strata of THpositive processes (Fig. 1j). DSCAM immunoreactivity was absent
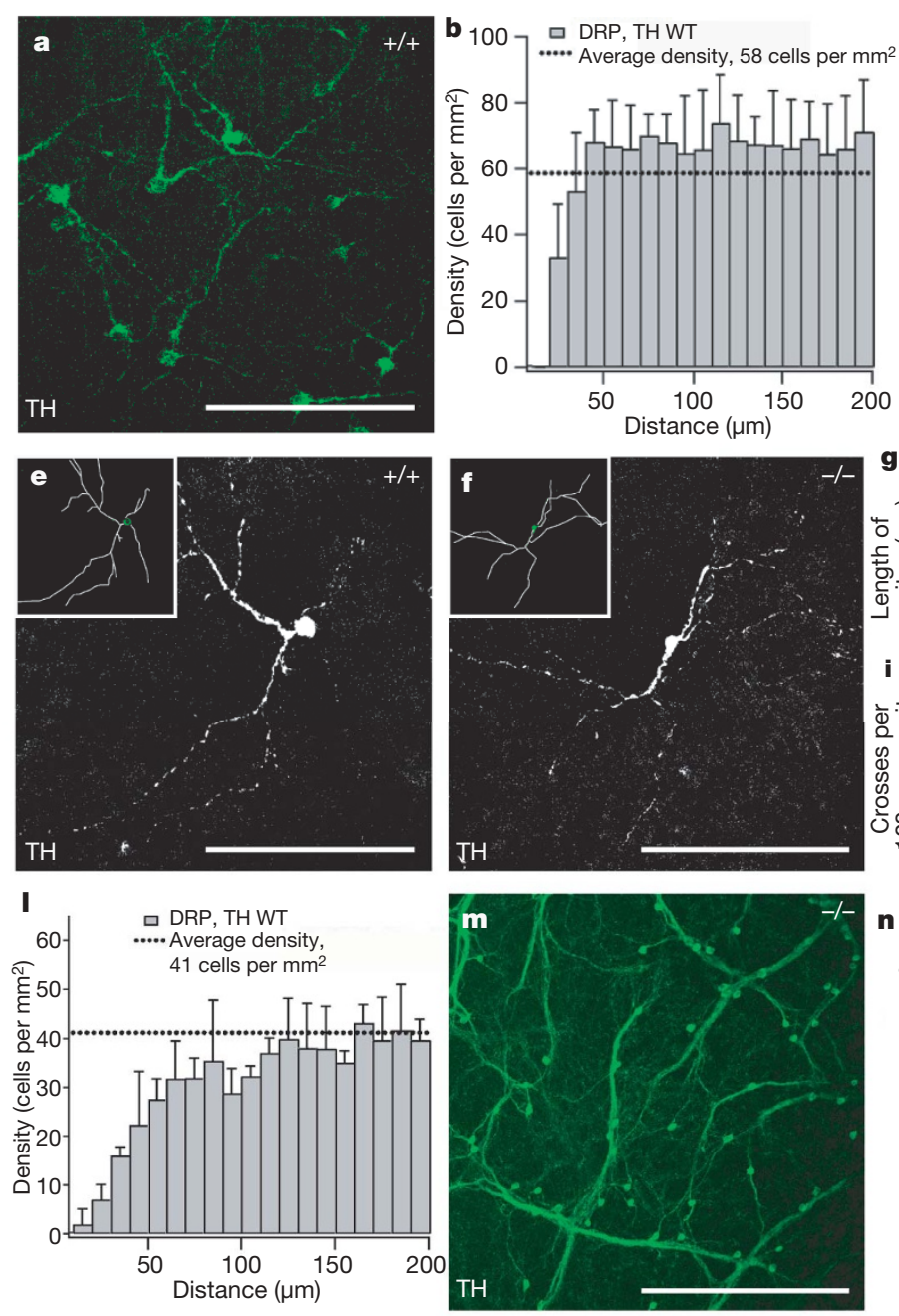

Figure 2 | Arborization and mosaic patterning of dopaminergic amacrine cells in control and Dscam ${ }^{-1-}$ retinas. a, A wild-type P6 retina stained with anti-TH. Dopaminergic amacrine cell processes have little proximal contact and rarely self-cross. $\mathbf{b}$, DRP analysis of cell-body spacing identified an exclusion zone surrounding dopaminergic neurons in wild-type retinas. c, A P6 Dscam ${ }^{-1-}$ retina stained with anti-TH. Individual dopaminergic amacrine cells frequently self-cross. Error bars in all figures represent s.d. d, DRP analysis of Dscam ${ }^{-/-}$dopaminergic amacrine cells indicates normal soma spacing at P6. e, f, Representative isolated P6 wild-type (e) and

Dscam $^{-1-}$ (f) dopaminergic amacrine cells are shown; insets contain traces of the neurites. $\mathbf{g}-\mathbf{j}$, Analysis of 20 isolated dopaminergic amacrine neurons from either wild-type or Dscam ${ }^{-/-}$retinas. $g$, No significant difference in the total neurite length was observed between wild-type and $\mathrm{Dscam}^{-/-}$neurons ( $t$-test, $P=0.55) . \mathbf{h}$, Similarly, no significant difference in neurite branches per unit length was observed $(P=0.38)$. $\mathbf{i}, \mathbf{j}$, A significant increase in the number of self-crossings per length of neurite (i) or per number of branches
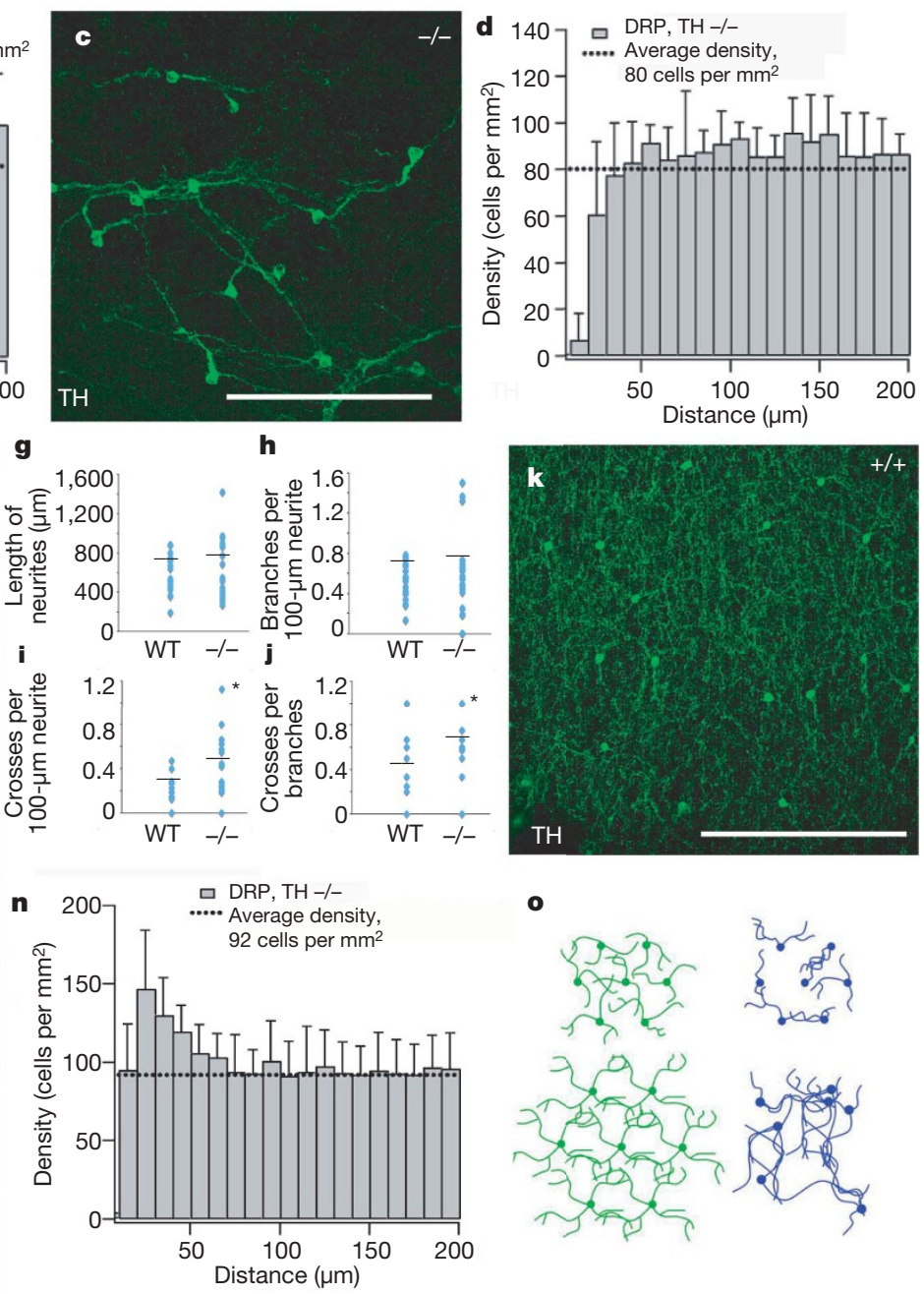

o

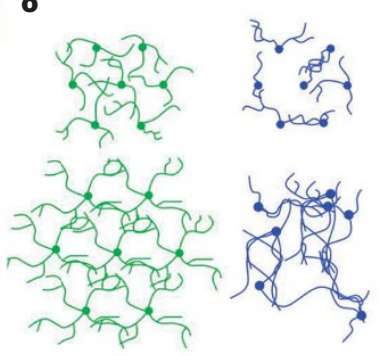

(j) was observed for the Dscam ${ }^{-/-}$neurons over controls $(P=0.003$ and 0.015 , respectively). $\mathbf{k}-\mathbf{n}$, Whole adult (6-8 weeks) wild-type (k, I) or

$\operatorname{Dscam}^{-/-}(\mathbf{m}, \mathbf{n})$ retinas stained with anti-TH. TH-positive neurite fascicles run throughout the Dscam ${ }^{-/-}$retina $(\mathbf{m})$ in contrast to the wild-type retina (k), in which neurites arborize evenly. I, DRP analysis indicates that dopaminergic amacrine cells in the adult wild-type retina maintain and expand their mosaic spacing and exclusion zones. n, DRP analysis of adult Dscam $^{-1-}$ retinas indicates aggregation of dopaminergic amacrine neurons. o, Schematic of arborization and mosaic formation in wild-type (left) and Dscam $^{-1-}$ (right) retinas. At P6, both wild-type and Dscam dopaminergic amacrine cells are organized in a mosaic pattern; however, wild-type neurites arborize, whereas Dscam $^{-1-}$ neurites self-cross. By P10, wild-type dopaminergic amacrine cells have an increased exclusion zone, whereas Dscam $^{-1-}$ dopaminergic amacrine neurons aggregate along bundled fascicles. Scale bars: a, c, e, f, $133 \mu \mathrm{m} ; \mathbf{k}, \mathbf{m}, 388 \mu \mathrm{m}$. 
from the mutant retinas, consistent with a protein-null mutation (Fig. 1k).

Wild-type dopaminergic amacrine cells were first labelled by antiTH staining at around P6, when the neurons are still extending processes (Fig. 2a). The spacing of dopaminergic amacrine cell bodies was analysed by density recovery profiling (DRP) - a statistical measure of the cells' spatial autocorrelation, measuring the probability of encountering a homotypic cell body at varied distances from the reference cell. Regularly spaced cells have a zone of exclusion for other homotypic cells, indicated by a below-average cell density at short distances ${ }^{15}$ (Fig. 2b). Although their spacing was normal, the Dscam $^{-1-}$ dopaminergic amacrine cells showed abnormal morphology at P6 (Fig. 2c, d). Examining individual cell morphologies in 20 isolated dopaminergic amacrine cells in control and Dscam ${ }^{-1-}$ retinas revealed defects in arborization in the mutant retina (Fig. 2e, f). Quantification indicated no differences in total length of processes or in the number of branches per unit length, but mutant cells had a significantly larger number of processes that self-crossed
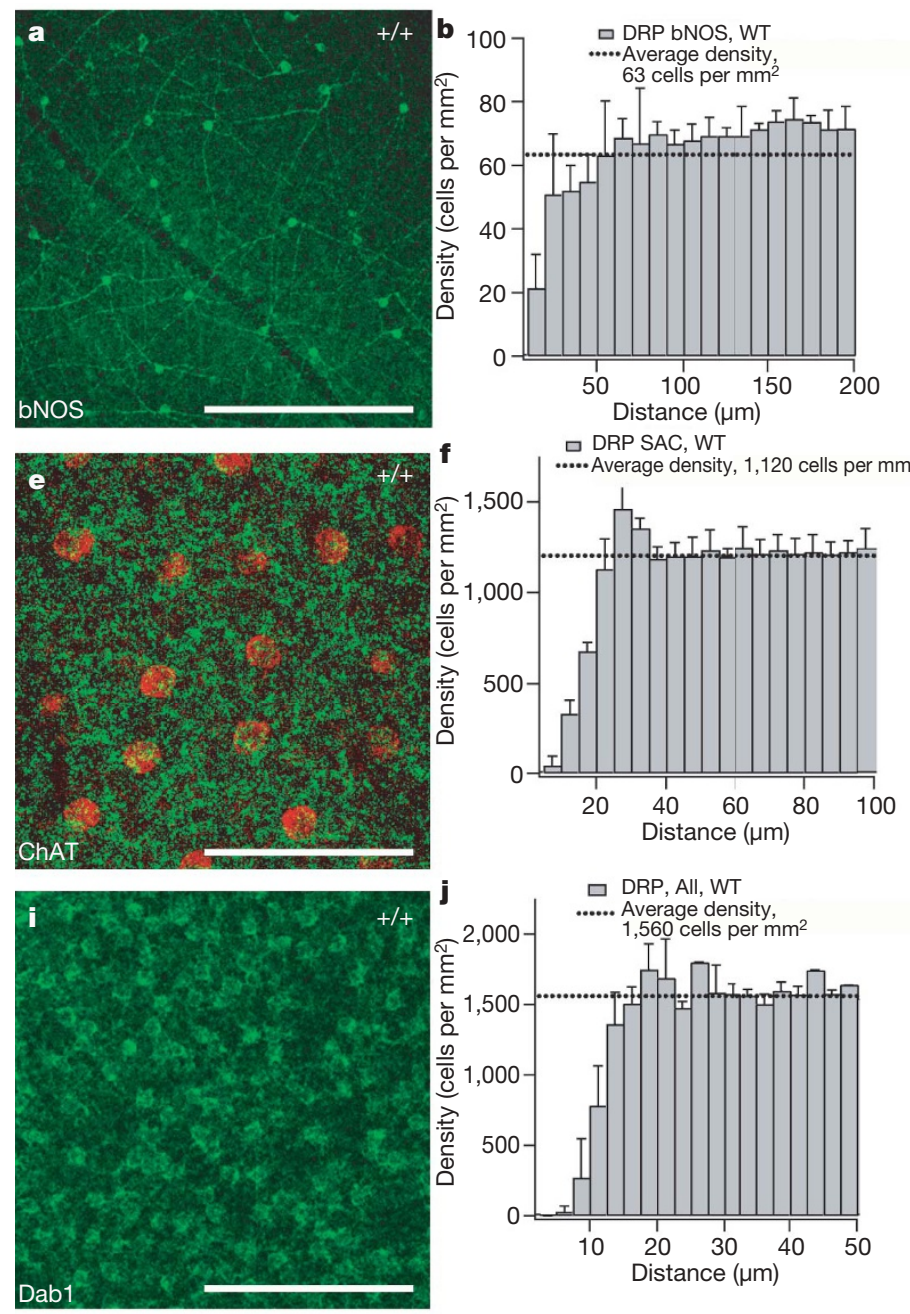

m

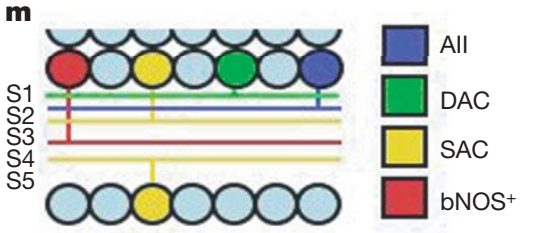

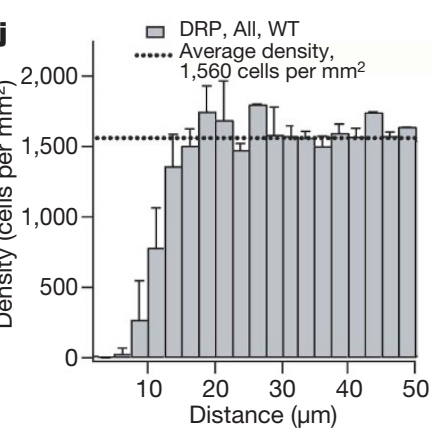

n
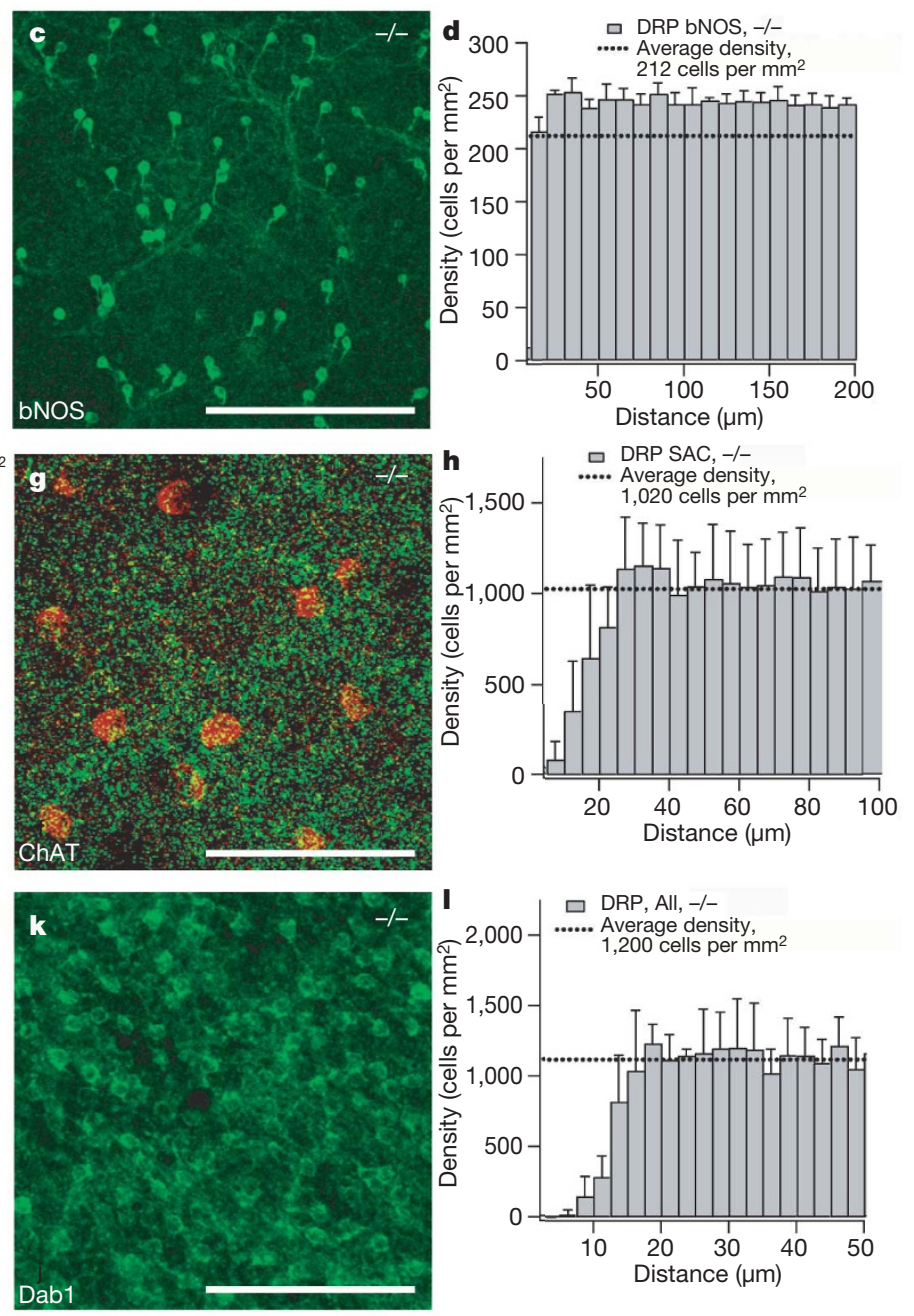

Dscam-positive cell type

Wild-type retina Dscam ${ }^{-/-}$retina
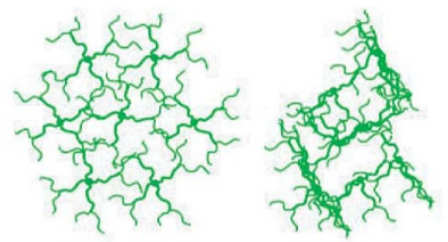

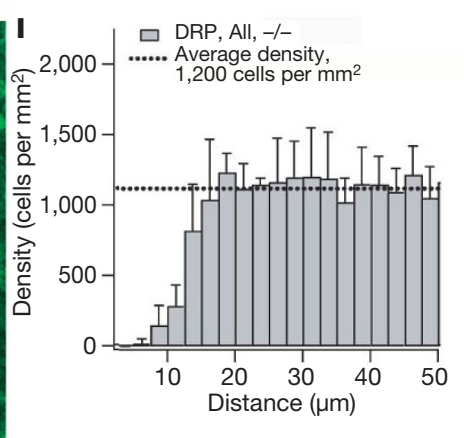

Dscam-negative cell type
Figure $\mathbf{3}$ | Morphology and cell body spacing of other amacrine cell types. a, b, Cell bodies of bNOS-positive cells, which also express Dscam in control retinas, are spaced and arborized in a manner similar to dopaminergic amacrine neurons. c, d, The bNOS-positive amacrine cells show fasciculated processes and randomly spaced cell bodies in $\mathrm{Dscam}^{-1-}$ retinas. Starburst amacrine cells have an exclusion zone around the cell body and a meshwork of arborized processes in both control $(\mathbf{e}, \mathbf{f})$ and $\operatorname{Dscam}^{-1-}(\mathbf{g}, \mathbf{h})$ retinas. (Cell bodies are pseudo-colored red to distinguish them from the meshwork of processes in S2 of the IPL (green).) AII amacrine cells are also evenly spaced with very short processes in control $(\mathbf{i}, \mathbf{j})$ and $\operatorname{Dscam}^{-/-}(\mathbf{k}, \mathbf{I})$ retinas. $\mathbf{m}$, Schematic depicting the stratification of bNOS-positive, SAC, DAC and
AII neurites in the inner plexiform layer. Dopaminergic amacrine neurites arborize predominantly in S1, AII amacrine neurites arborize in between $S 1$ and S2, bNOS-positive amacrine neurites arborize in S3, and starburst amacrine neurites arborize in S2 (soma in INL) or S4 (soma in RGL). n, Schematic summarizing mosaic formation in the $\mathrm{Dscam}^{-1-}$ retina. DSCAM-positive cell types arborize and maintain mosaics in the wild-type retina, but fasciculate and fail to maintain mosaics in the $D s c a m^{-1-}$ retina. Cell types in which DSCAM expression was not detected arborize and maintain mosaics in both the wild type and the $D$ scam ${ }^{-1-}$ retina. Scale bars: a, c, $388 \mu \mathrm{m}$; e, g, $62 \mu \mathrm{m}$; i, k, $123 \mu \mathrm{m}$. Different scales are used on the ordinates of DRP graphs for each cell type. 
(Fig. 2g-j). This phenotype was more pronounced at P10 (not shown), and eventually large fascicles of TH-positive processes from many cells form in the mutant retina (Supplementary Fig. 6). Dopaminergic processes in the wild-type adult retina form a broad, uniform plexus, and the cell bodies are evenly spaced (Fig. $2 \mathrm{k}, 1$ ). The fasciculated adult $D_{\text {scam }}{ }^{-/}$dopaminergic amacrine cells had abnormal positioning of their cell bodies, which associated with the fascicles and clumped significantly (Fig. 2m, n and Supplementary Figs 6 and 7).

In the mutant retina, bNOS-positive amacrine cells, which normally express Dscam, also had marked differences in the cell morphology, with short bundled processes and randomly spaced cell bodies (Fig. 3a-d). Starburst and AII amacrine populations that do not express Dscam were normal in their cell-body distribution (Fig. $3 \mathrm{e}-1$ ), maintaining an exclusion zone in the control and Dscam $^{-1-}$ retinas. The meshwork of processes in the IPL from starburst cells in the inner nuclear layer was preserved in the $D s \mathrm{cam}^{-/-}$ retinas, with no abnormal fasciculation seen, and the short processes of AII cells were also normal. Cross-sections of control and $\mathrm{Dscam}^{-1-}$ retinas were also examined, and all cell types reached their appropriate vertical layer in the retina (Supplementary Fig. 8).

In normal retinas, the mosaic spacing of each cell type, such as AII and starburst cells, is independent, allowing dendritic overlap of functionally different cells ${ }^{16}$. If DSCAM-mediated recognition was required for this process, then cell types that express Dscam might influence the spacing of each other. We therefore examined the distribution of dopaminergic amacrine and bNOS-positive amacrine cells, relative to each other, in control and mutant retinas (Fig. 4 and Supplementary Fig. 9). These cell bodies were randomly spaced with respect to one another in both genotypes, indicating that DSCAM is necessary for maintaining mosaics in homotypic cells but is not sufficient to confer identifying signals between cell types. Also, despite the fasciculation of dopaminergic and bNOS-positive processes in the $\mathrm{Dscam}^{-/-}$retinas, no associations of processes across cell types were seen.

Mammalian DSCAM is therefore required for isoneuronal selfavoidance and for heteroneuronal recognition within a cell type. In the absence of DSCAM, the processes of an individual dopaminergic or bNOS-positive amacrine cell fail to form proper arbors, and instead fasciculate with processes of other cells of the same type, secondarily pulling the cell bodies out of their mosaic position. However, different cell types expressing Dscam do not influence each other's spacing. A neurodevelopmental role for vertebrate DSCAM was not previously established beyond an early, non-neuron-specific, developmental phenotype seen in zebrafish ${ }^{17}$.

Our results are consistent with previous studies showing that retinal mosaics form very early, before neurons have extensive arbors, and that arborization and mosaic formation are largely independent $^{18,19}$. The normal spacing of cells in the P6 Dscam $^{-/-}$retina indicates that the early stages of mosaic formation are intact. However, DSCAM-mediated heteroneuronal self-avoidance is needed to prevent fasciculation of homotypic cells, which destroys their mosaic pattern.

Decreased cell death may also indirectly contribute to the disruption of mosaics, based on the increase in dopaminergic and bNOS cell densities in the mutant retina. The absolute density of cells that would normally express Dscam was increased by $250 \%$ for dopaminergic amacrine cells, and by $\sim 300 \%$ for bNOS-positive cells in the mutant retina (DRPs in Figs 2 and 3). In contrast, the densities of the ChAT-positive (starburst) and DAB1-positive (AII) cells were slightly decreased. Dopaminergic amacrine cells rely in part on cell death to sculpt their mosaic pattern ${ }^{20}$. We saw decreased TdTmediated dUTP nick end labelling (TUNEL)-positive cells in the developing Dscam $^{-/-}$retina, but saw no change in the number of proliferating cells (Supplementary Fig. 10); we also identified that clumped dopaminergic amacrine cells were not clonal, indicating that they are not the result of overproliferation from a progenitor, but consistent with lateral migration of cell bodies (Supplementary Fig. 11). DSCAM-mediated self-recognition may therefore be upstream of cell death in the wild-type retina, whereas non-Dscamexpressing cells may use other mechanisms for spacing ${ }^{21}$.

The fact that DSCAM mediates both isoneuronal and heteroneuronal self-avoidance in given amacrine cell types is interesting given recent work in Drosophila. In flies, Dscam is alternatively spliced to generate tens of thousands of protein isoforms ${ }^{6}$. The homophilic interaction of DSCAM is highly isoform-specific, and this specificity is determined by the variable immunoglobulin domains ${ }^{8,22,23}$. Therefore, Drosophila neurons use DSCAM for isoneuronal self-avoidance and arborization because each cell recognizes only its own processes, which express the same set of isoforms ${ }^{8,9,24}$. Reducing or eliminating the diversity of DSCAM isoforms perturbs Drosophila circuit organization $^{25,26}$. Dscam2 in Drosophila is not extensively alternatively spliced and mediates heteroneuronal cell avoidance in tiling
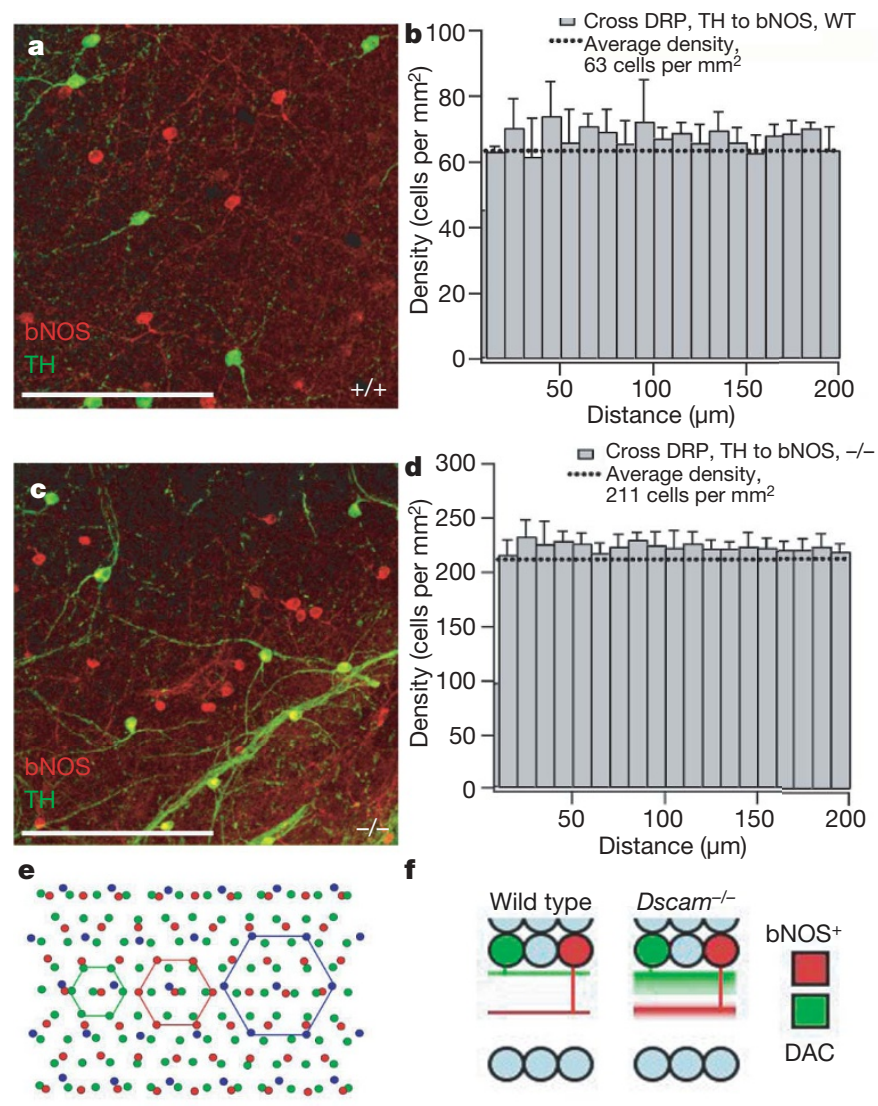

Figure 4 | Co-existence of dopaminergic and bNOS-positive amacrine cells. a, In wild-type retinas double-labelled for two Dscam-expressing cell populations (dopaminergic amacrine and bNOS-positive), the cell bodies and processes appear independent. $\mathbf{b}$, This was confirmed by cross-DRP analyses comparing dopaminergic to bNOS-positive cells, which indicated no difference from average density (random spacing). c, In Dscam ${ }^{-}$ mutant retinas, the fasciculation and cell-body-spacing defects are evident, but the spacing of cells remains independent and they do not aggregate, as indicated by cross-DRP analyses (d). In addition, the fascicles of processes also remain independent. e, Schematic depicting the independence of retinal neuron mosaic formation. Retinal neurons form organized mosaics that are independent of the spacing of other cell types. $f$, Schematic depicting dopaminergic amacrine and bNOS-positive neuron arborization in the wildtype and Dscam $^{-1-}$ retina. Wild-type dopaminergic amacrine neurons arborize in S1, whereas bNOS-positive neurons arborize in S3. In the Dscam $^{-1-}$ retina, the arborization of dopaminergic amacrine and bNOSpositive neurites becomes diffuse but, although neurites of both cell types fasciculate with homotypic neurites (dopaminergic amacrine with dopaminergic amacrine or bNOS-positive with bNOS-positive), they do not fasciculate with heterotypic neurites (dopaminergic amacrine with bNOSpositive). Scale bars in a and c represent $240 \mu \mathrm{m}$. 
the axons of L1 neurons in the medulla of the Drosophila visual system $^{7}$. Mouse DSCAM functions in a highly analogous way, promoting self-avoidance for both arborization (isoneuronal) and the prevention of fasciculation (heteroneuronal). Although the vertebrate genes are not extensively alternatively spliced, they do undergo homophilic and paralogue-specific binding ${ }^{5,27,28}$. The coexistence of Dscam-expressing amacrine cell populations suggests that stratification in different IPL layers may allow reuse of DSCAM as a recognition signal. Alternatively, additional co-signals may distinguish distinct cell populations, consistent with the continued lack of interaction between bNOS and dopaminergic cells in the Dscam $^{-/-}$retina. Additional complexity for self-recognition could also be introduced by other Dscam gene family members, such as Dscam-like 1 (Dscaml1), which is expressed in a similar but discrete population of retinal neurons ${ }^{27,28}$ (P.G.F. and R.W.B., unpublished) or the highly homologous Sidekick proteins ${ }^{29}$.

\section{METHODS SUMMARY}

Genetics and Dscam analysis. The Dscam mutation was mapped by breeding to C57BL6/J and establishing linkage in F2 mice with polymorphic markers, and the mutation was identified by positional cloning approaches. Mice were genotyped by PCR from genomic DNA spanning the 38-bp deletion (Dscam forward, CTTTGCGCGTTATGATCCT; Dscam reverse, GTGGTGTCGATACTGATG). Control mice were littermates of the $\mathrm{Dscam}^{-1-}$ animals to control for genetic background effects and age.

Immunocytochemistry. Whole-mount dissected retinas or cryostat sections were stained using standard immunofluorescence protocols. Antibody sources are listed in the Methods. Fluorescence images were collected using a Leica SP5 confocal microscope or a Nikon epifluorescence microscope with a digital camera.

In situ hybridization. In situ hybridization was performed using digoxygeninand fluorescein isothiocyanate (FITC)-labelled riboprobes, detected by HRPconjugated secondary antibodies and TSA-Plus fluorescent substrates (Perkin Elmer).

Analysis of mosaics. The spacing of cell bodies was analysed using DRP as described previously, with the modification that the closest bin in the analysis was corrected to account for the diameter of the cell body $y^{15,16}$.

Full Methods and any associated references are available in the online version of the paper at www.nature.com/nature.

\section{Received 2 October; accepted 21 November 2007.}

1. Wassle, H. \& Boycott, B. B. Functional architecture of the mammalian retina. Physiol. Rev. 71, 447-480 (1991).

2. Novelli, E., Resta, V. \& Galli-Resta, L. Mechanisms controlling the formation of retinal mosaics. Prog. Brain Res. 147, 141-153 (2005).

3. Galli-Resta, L. Local, possibly contact-mediated signalling restricted to homotypic neurons controls the regular spacing of cells within the cholinergic arrays in the developing rodent retina. Development 127, 1509-1516 (2000).

4. Yamakawa, K. et al. DSCAM: a novel member of the immunoglobulin superfamily maps in a Down syndrome region and is involved in the development of the nervous system. Hum. Mol. Genet. 7, 227-237 (1998).

5. Agarwala, K. L., Nakamura, S., Tsutsumi, Y. \& Yamakawa, K. Down syndrome cell adhesion molecule DSCAM mediates homophilic intercellular adhesion. Brain Res. Mol. Brain Res. 79, 118-126 (2000).

6. Schmucker, D. et al. Drosophila Dscam is an axon guidance receptor exhibiting extraordinary molecular diversity. Cell 101, 671-684 (2000).

7. Millard, S. S., Flanagan, J. J., Pappu, K. S., Wu, W. \& Zipursky, S. L. Dscam2 mediates axonal tiling in the Drosophila visual system. Nature 447, 720-724 (2007).

8. Wojtowicz, W. M., Flanagan, J. J., Millard, S. S., Zipursky, S. L. \& Clemens, J. C. Alternative splicing of Drosophila Dscam generates axon guidance receptors that exhibit isoform-specific homophilic binding. Cell 118, 619-633 (2004).
9. Zhan, X. L. et al. Analysis of Dscam diversity in regulating axon guidance in Drosophila mushroom bodies. Neuron 43, 673-686 (2004).

10. Soba, P. et al. Drosophila sensory neurons require Dscam for dendritic selfavoidance and proper dendritic field organization. Neuron 54, 403-416 (2007)

11. Matthews, B. J. et al. Dendrite self-avoidance is controlled by Dscam. Cell 129, 593-604 (2007).

12. Hughes, M. E. et al. Homophilic Dscam interactions control complex dendrite morphogenesis. Neuron 54, 417-427 (2007).

13. Jeon, C. J., Strettoi, E. \& Masland, R. H. The major cell populations of the mouse retina. J. Neurosci. 18, 8936-8946 (1998).

14. Kong, J. H., Fish, D. R., Rockhill, R. L. \& Masland, R. H. Diversity of ganglion cells in the mouse retina: unsupervised morphological classification and its limits. J. Comp. Neurol. 489, 293-310 (2005).

15. Rodieck, R. W. The density recovery profile: a method for the analysis of points in the plane applicable to retinal studies. Vis. Neurosci. 6, 95-111 (1991).

16. Rockhill, R. L., Euler, T. \& Masland, R. H. Spatial order within but not between types of retinal neurons. Proc. Natl Acad. Sci. USA 97, 2303-2307 (2000).

17. Yimlamai, D., Konnikova, L., Moss, L. G. \& Jay, D. G. The zebrafish Down syndrome cell adhesion molecule is involved in cell movement during embryogenesis. Dev. Biol. 279, 44-57 (2005).

18. Galli-Resta, L., Resta, G., Tan, S. S. \& Reese, B. E. Mosaics of islet-1-expressing amacrine cells assembled by short-range cellular interactions. J. Neurosci. 17, 7831-7838 (1997).

19. Lin, B., Wang, S. W. \& Masland, R. H. Retinal ganglion cell type, size, and spacing can be specified independent of homotypic dendritic contacts. Neuron 43 , 475-485 (2004).

20. Raven, M. A., Eglen, S. J., Ohab, J. J. \& Reese, B. E. Determinants of the exclusion zone in dopaminergic amacrine cell mosaics. J. Comp. Neurol. 461, 123-136 (2003).

21. Resta, V., Novelli, E., Di Virgilio, F. \& Galli-Resta, L. Neuronal death induced by endogenous extracellular ATP in retinal cholinergic neuron density control. Development 132, 2873-2882 (2005).

22. Wojtowicz, W. M. et al. A vast repertoire of Dscam binding specificities arises from modular interactions of variable Ig domains. Cell 130, 1134-1145 (2007).

23. Meijers, R. et al. Structural basis of Dscam isoform specificity. Nature 449, 487-491 (2007).

24. Neves, G., Zucker, J., Daly, M. \& Chess, A. Stochastic yet biased expression of multiple Dscam splice variants by individual cells. Nature Genet. 36, 240-246 (2004).

25. Hattori, D. et al. Dscam diversity is essential for neuronal wiring and selfrecognition. Nature 449, 223-227 (2007).

26. Chen, B. E. et al. The molecular diversity of Dscam is functionally required for neuronal wiring specificity in Drosophila. Cell 125, 607-620 (2006).

27. Agarwala, K. L. et al. Cloning and functional characterization of DSCAML1, a novel DSCAM-like cell adhesion molecule that mediates homophilic intercellular adhesion. Biochem. Biophys. Res. Commun. 285, 760-772 (2001).

28. Yamagata, M. \& Sanes, J. R. Dscam and Sidekick proteins direct lamina-specific synaptic connections in vertebrate retina. Nature doi:10.1038/nature06469 (this issue).

29. Yamagata, M., Weiner, J. A. \& Sanes, J. R. Sidekicks: synaptic adhesion molecules that promote lamina-specific connectivity in the retina. Cell 110, 649-660 (2002).

Supplementary Information is linked to the online version of the paper at www.nature.com/nature.

Acknowledgements We thank G. Cox and S. Ackerman for comments on the manuscript, and M. Yamagata and J. Sanes for sharing unpublished results. The authors are supported by the NEI, the Knights Templar Eye Foundation (P.G.F.) and Research to Prevent Blindness (R.H.M.). We also thank the scientific services at The Jackson Laboratory (supported by NCl Cancer Center).

Author Contributions P.G.F. identified the Dscam mutation and performed all tissue staining and microscopy presented. A.K. carried out the quantitative analyses of retinal cell mosaics and microinjected TH cells. R.H.M. helped to interpret the anatomical images and designed the mosaic analysis. R.W.B. assisted in experimental design and interpretation. All authors shared in writing the paper.

Author Information Reprints and permissions information is available at www.nature.com/reprints. Correspondence and requests for materials should be addressed to R.W.B. (robert.burgess@jax.org). 


\section{METHODS}

Animal husbandry. Mice were used in accordance with protocols approved by the Animal Care and Use Committee at the Jackson Laboratory. The Jackson Laboratory is accredited by the Association for the Assessment and Accreditation or Laboratory Animal Care (AAALAC). Animals were housed in PIV caging and given food and water ad libitum.

Genetic mapping. The Dscam ${ }^{\text {del17 }}$ mutation arose spontaneously in the Balb/cJ mapping partner of an unrelated F1 mapping cross. The mutation was initially localized to the distal region of chromosome 16 by typing 140 strain-specific markers, spaced approximately every $10 \mathrm{Mb}$, on a total of 10 mutant and 10 wildtype mice, the phenotypes of which were confirmed by retinal histology. The mutation was fine-mapped against DBA/2J, C57BL/6J and CAST/EiJ strains to maximize the number of polymorphic markers available. In total, more than 1,800 meioses were typed at the flanking markers D16Mit106 and D16Mit94. The interval was narrowed to $1.71 \mathrm{Mb}(0.2 \mathrm{cM})$, between D16Mit219 and D16Mit205. This interval contained 14 annotated genes including mouse Dscam. For analysis of the Dscam gene, mRNA from affected and control brain was prepared using Trizol (Invitrogen), reverse transcribed with SuperscriptIII (Invitrogen) for PCR and sequencing, or used for northern blot analysis.

Mouse strains. The official strain designation of the Dscam mutation is Dscam $^{\text {del17 }}$, reflecting the molecular lesion. It is referred to in this paper as $\mathrm{Dscam}^{-}$. In segregating genetic backgrounds containing BALB/c or 129 loci, these mice survive to adulthood and will breed as homozygotes. However, as the background is made increasingly $\mathrm{C} 57 \mathrm{BL} / 6$, the affected mice die neonatally. The experiments described were therefore carried out on a mixed, but predominantly BALB/cByJ, background. Littermates of experimental mutants were used as controls whenever possible to account for age and genetic background. The yellow fluorescent protein (YFP) transgenic strain used in Supplementary Fig. 6 is the MitoZ strain.

Genotyping. The primers Dscam17F and Dscam17R (CTTTGCGCGTTATGATCCT and GTGGTGTCGATACTGATG, respectively), which flank the Dscam $^{\text {del17 }}$ deletion, were used to genotype mice. Tail biopsies were digested overnight in $100 \mu \mathrm{l}$ of $1 \mathrm{mg} \mathrm{ml}^{-1}$ proteinase-K in buffer containing $100 \mathrm{mM}$ Tris, pH 8.5, $10 \mathrm{mM}$ EDTA and $200 \mathrm{mM} \mathrm{NaCl}$. Samples were then boiled for $15 \mathrm{~min}$ to denature the DNA and to inactivate the proteinase-K. PCR was performed using Eppendorf master taq with the following program: $94^{\circ} \mathrm{C}$ for $1 \mathrm{~min}$, followed by $35 \mathrm{cycles}$ of $94^{\circ} \mathrm{C}$ for $20 \mathrm{sec}, 53^{\circ} \mathrm{C}$ for $30 \mathrm{sec}$ and $72^{\circ} \mathrm{C}$ for $30 \mathrm{sec}$, followed by a single $72^{\circ} \mathrm{C}$ extension for $2 \mathrm{~min}$. PCR of DNA from homozygous wild-type mice amplifies a single $170 \mathrm{bp}$ band, whereas PCR of DNA from homozygous mutant mice have a single $133 \mathrm{bp}$ band, with both bands apparent in PCR samples run using DNA from heterozygous mice.

Analysis of mRNA. For RNA isolation, total retinal or brain RNA was isolated by dissecting the retina or brain of wild-type and $\mathrm{Dscam}^{-/-}$mice, which were then homogenized in $500 \mu \mathrm{l}$ or $2 \mathrm{ml}$ Trizol reagent, respectively, according to the manufacturer's protocol. RNA samples were resuspended in diethyl pyrocarbonate (DEPC)-treated $\mathrm{H}_{2} \mathrm{O}$. Reverse transcription was performed using SuperScript First-Strand Synthesis kit according to manufacturer's instructions using Superscript III reverse transcriptase and a mix of random hexamer and oligo-dT primers (Invitrogen). For northern blot analysis, a probe to the aminoterminal region of the Dscam transcript was generated by PCR with reverse transcription (RT-PCR) with primers Dscam1 forward and Dscam1 reverse (TCCTTCAAGCTTCAGCAC and CATTTCCTGTCACACTGC, respectively). This fragment was labelled with ${ }^{32} \mathrm{P}$ using the Amersham Rediprime II Random labelling kit according to the manufacturer's instructions (GE Healthcare). Total RNA $(25 \mu \mathrm{g})$ from each sample was heated at $75^{\circ} \mathrm{C}$ for $5 \mathrm{~min}$ and loaded onto a $2 \%$ formaldehyde gel. Electrophoresis of samples was conducted for $5 \mathrm{~h}$ at 50 volts $(\mathrm{V})$. RNA was transferred to a charged nylon membrane for 16-18 hours using the standard sandwich technique. RNA was crosslinked to the membrane with ultraviolet light and prehybridized at $50{ }^{\circ} \mathrm{C}$ in $10 \mathrm{ml}$ hybridization buffer for $1-2$ hours $(5 \times$ SSC, $1 \times$ Denhardt's solution, $1 \%$ salmon sperm DNA $\left(100 \mu \mathrm{g} \mathrm{ml}^{-1}\right), 50 \%$ formamide and $0.5 \%$ SDS). Labelled probe $(10 \mu \mathrm{Ci})$ was boiled in TE $(10 \mathrm{mM}$ Tris, $\mathrm{pH} 7.5$, and $1 \mathrm{mM}$ EDTA) for $5 \mathrm{~min}$ to denature and was added to $5 \mathrm{ml}$ of hybridization buffer. Blots were hybridized at $65^{\circ} \mathrm{C}$ for $16-18$ hours. Blots were washed two times for $15 \mathrm{~min}$ in $2 \times$ SSC and $0.5 \%$ SDS followed by two 15 -min washes in $0.2 \times$ SSC and $0.1 \%$ SDS at $65^{\circ} \mathrm{C}$. Transcript levels were compared in three Dscam ${ }^{-1-}$ mice, three Dscam $^{+/-}$mice and three Dscam $^{+/+}$mice at ten days of age.

RNA in situ hybridization. Probe templates were prepared by PCR from firststrand brain cDNA. The primers contained T7 (anti-sense) or SP6 (sense) transcription initiation sequences at the $3^{\prime}$ and $5^{\prime}$ ends, respectively, of the PCR products. RNA probes labelled with digoxygenin or fluorescein dUTP were prepared using the following reaction mixture: $1 \mu \mathrm{g}$ DNA, $2 \mu \mathrm{l} 10 \times$ T7 or SP6 buffer (Roche), $1.5 \mu \mathrm{l}$ T7 or SP6 RNA polymerase (Roche), $0.5 \mu$ l RNase out
(Invitrogen), $2 \mu$ ligoxyigenin or fluorescein dNTP mixture (Roche), and $\mathrm{H}_{2} \mathrm{O}$ to $20 \mu \mathrm{l}$. Samples were incubated at $37^{\circ} \mathrm{C}$ for $2 \mathrm{~h}$, after which $1 \mu \mathrm{l}$ of RNase-free DNase I (Roche) was added. Samples were incubated for an additional $15 \mathrm{~min}$, after which $1 \mu \mathrm{l} 0.5 \mathrm{M}$ EDTA was added to stop the reaction. The RNA was precipitated by adding $2.5 \mu \mathrm{l}$ of $4 \mathrm{M} \mathrm{NH}_{4} \mathrm{Cl}$ and $75 \mu \mathrm{l}$ of chilled $\left(-20^{\circ} \mathrm{C}\right)$ ethanol, and resuspended in $40 \mu \mathrm{l}$ of DEPC-treated $\mathrm{H}_{2} \mathrm{O}$. Typical yields were 300$400 \mathrm{ng} \mu \mathrm{l}^{-1}$ of labelled riboprobe.

For hybridization, eyes were enucleated and fixed in $4 \%$ paraformaldehyde at $4{ }^{\circ} \mathrm{C}$ for $4 \mathrm{~h}$ and were frozen in cyro-embedding media. Sections were cut at $15-$ $20 \mu \mathrm{m}$ with a cryostat and incubated at $65^{\circ} \mathrm{C}$ for $10 \mathrm{~min}$. Sections were again fixed with $4 \%$ paraformaldehyde for $10 \mathrm{~min}$ at room temperature $\left(20^{\circ} \mathrm{C}\right)$, followed by three washes with DEPC-treated PBS. Sections were digested with proteinase- $\mathrm{K}$ for $5 \mathrm{~min}$ at room temperature $\left(1 \mu \mathrm{g} \mathrm{ml}^{-1}\right.$ proteinase- $\mathrm{K}$ in $50 \mathrm{mM}$ Tris, $\mathrm{pH} 7.5$, and $5 \mathrm{mM}$ EDTA). After digestion, samples were fixed again in $4 \%$ paraformaldehyde for $5 \mathrm{~min}$ at room temperature. Sections were washed three times in DEPC-treated PBS. Sections were then acetylated for $10 \mathrm{~min}$ at room temperature $\left(59 \mathrm{ml} \mathrm{H} \mathrm{H}_{2} \mathrm{O}, 0.8 \mathrm{ml}\right.$ triethanolamine, $0.105 \mathrm{ml} \mathrm{HCl}$ and $0.15 \mathrm{ml}$ acetic anhydride). After acetylation, sections were permeabilized in $1 \%$ Triton X-100/PBS for $30 \mathrm{~min}$ at room temperature, followed by three $10-\mathrm{min}$ rinses in DEPC-treated PBS. Slides were prehybridized for one hour at room temperature in hybridization solution $(50 \%$ formamide, $5 \times$ SSC, $5 \times$ Denhart's solution, $250 \mu \mathrm{g} \mathrm{ml}^{-1}$ yeast tRNA, $500 \mu \mathrm{g}$ salmon sperm DNA and $50 \mu \mathrm{g} \mathrm{ml}^{-1}$ heparin). While sections were prehybridizing, probes were prepared at a concentration of $200 \mathrm{ng} \mathrm{ml}^{-1}$ in hybridization solution. Probe solution $(50 \mu \mathrm{l})$ was prepared per slide and incubated at $80^{\circ} \mathrm{C}$ for $5-10 \mathrm{~min}$ to denature. Prehybridization solution was replaced with hybridization solution, and the slides were coverslipped with 50-mm glass coverslips and incubated in a humidified chamber ( $50 \%$ formamide $5 \times$ SSC) at $65-70^{\circ} \mathrm{C}$ overnight.

For probe detection, after hybridization, sections were washed twice for $5 \mathrm{~min}$ with TBS at room temperature. Peroxidase-conjugated antibodies to digoxygenin or fluorescein (Roche) were diluted 1:2,000 in TNB (TSA plus system) and incubated on sections at $4{ }^{\circ} \mathrm{C}$ overnight. Sections were washed in TBST $(0.05 \%$ Tween) three times for $10 \mathrm{~min}$ at room temperature. Probes were detected using the TSA plus system (Perkin-Elmer). For double-labelling experiments, POD-antibodies against FITC and digoxygenin (DIG) were applied sequentially, and the peroxidase conjugate of the first detection antibody was inactivated by $\mathrm{H}_{2} \mathrm{O}_{2}$ treatment before applying the second antibody. Different tyramide conjugates were used when developing the signals to achieve different colours, and detection reactions were allowed to proceed for $30 \mathrm{~min}$. Dscam and amacrine-marker expression was examined at P12. At least four independent retinas were examined. Sense-strand controls were used in all experiments to confirm specificity.

Histology and immunocytochemistry. Tissues were fixed in Bouin's fixative overnight. Sections $(4 \mu \mathrm{m})$ were cut with a microtome and stained using a standard haemotoxylin and eosin stain. For immunocytochemistry, embryos or enucleated eyes were fixed in $4 \%$ paraformaldehyde overnight. Tissue was equilibrated in 30\% sucrose/PBS and frozen in OCT media. Thicker sections $(8 \mu \mathrm{m})$ were cut using a cryostat and used immediately or stored for up to two weeks at $-20{ }^{\circ} \mathrm{C}$. Sections were blocked in $3 \%$ normal horse serum in $1 \times$ PBS and $0.1 \%$ Triton X-100. Primary antibodies (indicated in the Methods Summary) were incubated overnight at $4{ }^{\circ} \mathrm{C}$ and subsequently washed twice for $10 \mathrm{~min}$ in PBS. Sections were incubated at room temperature with secondary antibodies for one hour, and then washed four times in PBS for $10 \mathrm{~min}$. The last wash contained $2 \mu \mathrm{l}$ of $1 \mathrm{mg} \mathrm{ml}^{-1}$ DAPI per $40 \mathrm{ml}$ PBS.

Whole-mounted retinas. Mice were perfused transcardially with PBS and 4\% paraformaldehyde. Eyes were enucleated and placed in a dish containing PBS. A small hole was made at the junction between the cilliary body and retina with a 30-gauge needle. The eye was hemisected and the retina was gently teased away from the sclera. Retinas were fixed a second time in $4 \%$ paraformaldehyde for onehour, followed by a rinse in PBS. Retinas were incubated with primary antibodies in PBS, supplemented with 3\% normal horse serum and $0.5 \%$ Triton $\mathrm{X}-100$, for $3-5$ days at $4{ }^{\circ} \mathrm{C}$ with gentle rocking. Primary antibodies were washed off overnight in PBS, and the retinas were incubated with secondary antibodies in PBS, 3\% normal horse serum and $0.5 \%$ Triton. Retinas were washed for $2-4 \mathrm{~h}$ in PBS and were flat-mounted. Whole-mount images were collected from mice between six and ten weeks of age, and at least six mutant and control animals were included in each analysis.

Antibodies used. The following primary antibodies were used in standard immunofluorescence and western blotting protocols. Goat anti-human DSCAM (R\&D Systems), rabbit anti-tyrosine hydroxylase (Novus Biologicals), goat anti-choline acetyltransferase (Chemicon), rabbit antiDisabled1 (gift from B. Howell), anti-CHX10 (Santa Cruz Biotechnology), anti-BRN3b (Santa Cruz Biotechnology), mouse anti-neurofilament (2H3, Developmental Studies Hybridoma Bank), mouse anti-PAX6 (Developmental 
Studies Hybridoma Bank), rabbit anti-bNOS (Sigma-Aldrich), mouse anti-TH (Novocastro), and mouse anti-Ki67 (Dako).

Western blot analysis. A P0 retina was dissected and homogenized in hypertonic sucrose lysis buffer $\left(0.32 \mathrm{M}\right.$ sucrose, $1 \mathrm{mM} \mathrm{NaHCO}_{3}, 1 \mathrm{mM} \mathrm{MgCl}_{2}$ and $0.5 \mathrm{mM}$ $\mathrm{CaCl}_{2}$ ) containing protease inhibitors with a glass dounce homogenizer. Total protein $(40 \mu \mathrm{g})$ was loaded onto $6 \%$ acrylamide gels, run and transferred. DSCAM antibodies were used at a concentration of 1:500. The specificity of the DSCAM antibody in western blotting was established using COS-7 cells transfected with c-Myc-tagged Dscam cDNA. A single band of approximately $220 \mathrm{kD}$ was observed when using an antibody to either c-Myc or DSCAM in the transfected sample only. Western blots from tissue were performed using the DSCAM antibody after pre-absorption against mutant tissue to reduce background staining. As a result, immunoreactivity to truncated protein products specific to the mutant could have been missed. The serum used in Fig. 1 for immunocytochemistry was not pre-absorbed.

Cell morphologies. P6 retinas were collected and stained with anti-TH as described previously. TH-positive amacrine cells that were sufficiently isolated from neighbouring cells to allow their processes to be definitively identified were imaged using a $\times 63$ objective lens on a Leica SP 5 confocal microscope. The total length of 20 wild-type and $20 \mathrm{Dscam}^{-1-}$ dendrites was traced, and measured using ImageJ.

DRP and nearest neighbour analysis analysis. DRP and nearest neighbour analysis (NNA) were performed as described previously ${ }^{23,24}$. The entire retinas were imaged and reconstructed for analysis of TH and bNOS amacrine cells. Four $775-\mu \mathrm{m}$ fields for each wild type and $\mathrm{Dscam}^{-1-}$ retina were imaged and used to analyse starburst and AII amacrine cells. Cholinergic amacrine cells were imaged in the inner nuclear layer for analysis. Independent samples from at least three animals, ages P6 and six to ten weeks, were included in each analysis.

TUNEL staining and analysis. TUNEL cell death detection kit was used to perform TUNEL analysis according to the manufacturer's instructions for difficult tissues (Roche). Eyes were enucleated and immediately frozen and cut in $10-\mu \mathrm{m}$ sections using a cryostat. After finishing the TUNEL reaction, the slides were washed in PBS for $5 \mathrm{~min}$, and immunocytochemistry was performed using a PAX6 antibody as detailed previously. TUNEL-positive cells were counted according to their location: retinal ganglion layer (including the inner plexiform layer), the inner neuroblast layer and the outer neuroblast layer. The inner and outer neuroblast layers were distinguished by PAX6 immunoreactivity. At least three slides with eight sections on each slide for each of three mice were recorded at each time point for each genotype.

Mitotic index. The mitotic index in the wild-type and $\mathrm{Dscam}^{-1-}$ retina was determined at three time points, E14, E17 and P1. BrDU injections were administered at a dosage of $10 \mathrm{mg} \mathrm{kg}^{-1} 20 \mathrm{~min}$ before tissue collections. Embryo heads or enucleated eyes were fixed overnight in $4 \%$ paraformaldehyde and were imbedded in paraffin blocks. Sections were cut at $5 \mu \mathrm{m}$ and stained with antibodies to BrDU and Ki67 after boiling in citrate buffer for $10 \mathrm{~min}$. The number of BrDU-positive/Ki67-positive cells (S-phase) were divided by the number of Ki67-positive cells (G2/M phase). Cells that did not label for either marker were also counted and no significant difference was observed in their number. At least three slides with eight sections on each slide for each of three mice were recorded at each time point for each genotype. 